\title{
Establishment of primary mouse lung adenocarcinoma cell culture
}

\author{
SHULI LUO ${ }^{1}$, MEI SUN ${ }^{2}$, RUI JIANG ${ }^{4}$, GUAN WANG $^{3}$ and XINYI ZHANG ${ }^{1}$ \\ Departments of ${ }^{1}$ Thoracic Surgery, ${ }^{2}$ Pathology and ${ }^{3}$ Cardiac Medicine, The Second Hospital of Jilin University, \\ Changchun 130041; ${ }^{4}$ Department of Orthopedic Surgery, China-Japan Union Hospital \\ of Jilin University, Changchun 130033, P.R. China
}

Received January 19, 2011; Accepted April 29, 2011

DOI: $10.3892 / 01.2011 .301$

\begin{abstract}
Lung cancer is the most common malignant disease worldwide and is the leading cause of death from cancer. Primary cultures derived from lung cancer are essential for understanding abnormal growth function in lung epithelia. In this study, 2 out of 5 primary lung adenocarcinoma cultures derived from DNA repair-deficient mice were established and characterised using electron microscopy and immunostaining. Results of the tumourigenicity tests confirmed that these primary cells are tumourigenic. In conclusion, an effective primary culture method provides a tool for clinical antitumour drug testing.
\end{abstract}

\section{Introduction}

Lung cancer is the most common malignant disease worldwide, and it is a major cause of death from cancer, particularly among males. Lung cancer almost exclusively involves carcinomas, arising from epithelial tissue of the trachea, bronchi or lung (1). Principal histological types of lung cancer are squamous cell carcinoma, adenocarcinoma, large cell carcinoma (collectively referred to as 'non-small cell' lung carcinomas) and small cell carcinoma (2). In addition to smoking, a positive familial history of lung cancer has been identified as a risk factor (3). Genetic alterations associated with lung cancer include frequent mutations in p53 gene, activating point mutations in the KRAS oncogene, frequent loss of heterozygosity and aberrant transcripts of the Fragile Histidine Triad (FHIT) gene, homozygous deletions and transcriptional silencing of the CDK inhibitor p16. Increased risk of lung cancer has been associated with certain polymorphisms of cytochrome P450 genes and with deficiencies in DNA repair capacity including DNA base excision repair genes XRCC1, PARP-1 and ERCC4 (4-6).

Correspondence to: Dr Xingyi Zhang, Department of Thoracic Surgery, The Second Hospital of Jilin University, 218 Ziqiang Street, Changchun 130041, P.R. China

E-mail: xyzhang@jlu.edu.cn

Key words: lung cancer, epithelial, primary culture, tumourigenicity
DNA damage response emerges as a biological tumourigenesis barrier in the early stages of cancer development, and exerts a selective pressure that favours outgrowth of malignant clones with defects in the genome maintenance machinery (7). Findings of previous studies showed that although wildtype mice rarely develop carcinomas, defective DNA repair in PARP-1 mutant mice leads to malignant epithelial tumours such as those of the lung, liver and mammary gland (8-10). To gain a better understanding of the role of DNA repair defects in lung carcinogenesis and to establish a primary culture method for anti-cancer drug screening primary mouse lung carcinoma cells were isolated from PARP-1 mutant mice.

\section{Materials and methods}

Primary cultures. For primary cell-culture experiments, lung tumours obtained from PARP-1 deficient mice (from the Jackson laboratory, Bar Harbor, ME, USA) at various ages were processed within $30 \mathrm{~min}$ after biopsy removal. Tumour specimens were cut into 1-mm slices in order that single cells as well as cell clumps (organoids) be released into the washing medium. Larger clumps were separated by allowing them to gravity settle for a few minutes. The clumps were reduced in size by gentle rotation in a test tube for 30-60 min. Cells and organoids isolated in this way were directly used for primary cultures, as described below. The remaining tissue slices were routinely subjected to enzymatic digestion with $0.3 \mathrm{mg} / \mathrm{ml}$ collagenase (Sigma, St. Louis, MO, USA) by gentle agitation for 1 to $2 \mathrm{~h}$ at $37^{\circ} \mathrm{C}$ in DMEM containing 5\% FCS, $200 \mathrm{U} / \mathrm{ml}$ penicillin, $200 \mu \mathrm{g} / \mathrm{ml}$ streptomycin, $50 \mu \mathrm{g} / \mathrm{ml}$ gentamycin and $50 \mathrm{U} / \mathrm{ml}$ nystatin. The procedure involved for the establishment of cell cultures was as previously described (11-13), and was adapted for the processing of mouse lung carcinoma.

Isolated cells and cell clumps were routinely seeded onto round glass coverslips in growth medium I containing $10 \%$ FCS , $4.0 \mathrm{mM}$ glutamine, $10 \mathrm{mM}$ HEPES, $100 \mathrm{U} / \mathrm{ml}$ penicillin, $100 \mu \mathrm{g} / \mathrm{ml}$ streptomycin, $50 \mu \mathrm{g} / \mathrm{ml}$ gentamycin, $50 \mathrm{U} / \mathrm{ml}$ nystatin, $1 \mu \mathrm{g} / \mathrm{ml}$ hydrocortisone (Sigma), $0.2 \mathrm{U} / \mathrm{ml}$ insulin (Sigma), $2 \mu \mathrm{g} / \mathrm{ml}$ transferring (Sigma) and $5 \mathrm{nM}$ sodium selenite (Sigma). After 1 week the medium was replaced by growth medium II (as above, without gentamycin and nystatin). Only those cultures in which fibroblast contamination was low, and in which overgrowth of epithelial cells by fibroblasts became unlikely, were processed further. Growth medium III was used (DMEM plus $10 \%$ heat-inactivated FCS, $50 \mathrm{U} / \mathrm{ml}$ penicillin, 
$50 \mu \mathrm{g} / \mathrm{ml}$ streptomycin, $4.0 \mathrm{mM}$ glutamine and $10 \mathrm{mM}$ HEPES). All cell culture media and reagents were purchased from Life Sciences Technologies (Blowing Rock, NC, USA).

Since fibroblast-conditioned medium appears to support growth of intestinal epithelial cells, preferential growth of epithelial cells without fibroblast overgrowth was initiated on glass coverslips by using mouse primary fibroblast cells (MPFs) lethally irradiated with 60 Gray (6,000 rads) of $\gamma$ radiation (IBL-437C, CIS Bio, Gif-Sur-Yvette, France). MPFs were seeded as a feeder layer in the tissue-culture plate on which glass coverslips were placed. Feeder layers were used at 30-40\% confluency and were maintained for 1 week. Alternatively, MPFs-culture supernatants were mixed 1:1 with fresh growth medium II (conditioned growth medium). For initial passaging, primary cultures were sub-cultured only when areas of tumour cell growth became confluent. For the first passage, all cells from a coverslip were mechanically scraped off and transferred to a fresh culture plate with conditioned growth medium. Fibroblast growth had to be continuously suppressed in cultures by using conditioned growth medium. Cellular morphology in the primary cultures was evaluated by light microscopy. The epithelial nature was characterised with electron microscopy and by immuostaining.

Electron microscopy and immunostaining. For scanning electron microscopy and transmission electron microscopy, as well as for immunostaining, cells were seeded on round glass coverslips. At the end of the culture time cells were washed in phosphate-buffered saline and fixed for $2 \mathrm{~h}$ in $2.5 \%$ glutaraldehyde in cacodylate buffer and processed by routine methods for electron microscopic examination. For immunostaining, cells were fixed with paraformaldhyde, incubated with monoclonal antibodies against pan-cytokeratins (NovoCastra, Newcastle, UK) and $\beta$-catenin (Cell Signaling, USA) or Cy3-conjugated anti-IgG antibody (Cappel, Organon, NC, USA). Glass coverslips were mounted upside-down in Vectashield mounting medium (Vector, Burlingame, CA, USA).

Tumourigenicity test. For the tumourigenicity test, cells were cultured to $70 \%$ confluence and trypsinised into single cell suspension. LC1 and LC2 $\left(1 \times 10^{6}\right)$ cells were inoculated subcutaneously into 5 immunocompromised nude mice $(\mathrm{Balb} / \mathrm{C})$ and maintained for two weeks. Tumours were collected and subjected to histological examination. The experimental protocols were approved by the Ethics Committee of the Second Bethune Hospital of Jilin University.

Histology and immunohistochemistry. Histological analysis was carried out on $3-\mu \mathrm{m}$ sections stained with haematoxylin and eosin, or immunostained (11-13). Antibodies included anti-pan-cytokeratin (NovoCastra) and E-cadherin (Chemicon International, Temecula, CA, USA).

\section{Results}

Primary cultures derived from mouse lung adenocarcinomas. By introducing modifications to previously published methods (13), i.e., using collagenase and shorter time periods for enzymatic digestion, as well as MPFs-conditioned growth medium for maintaining optimal growth of epithelial cells, viable
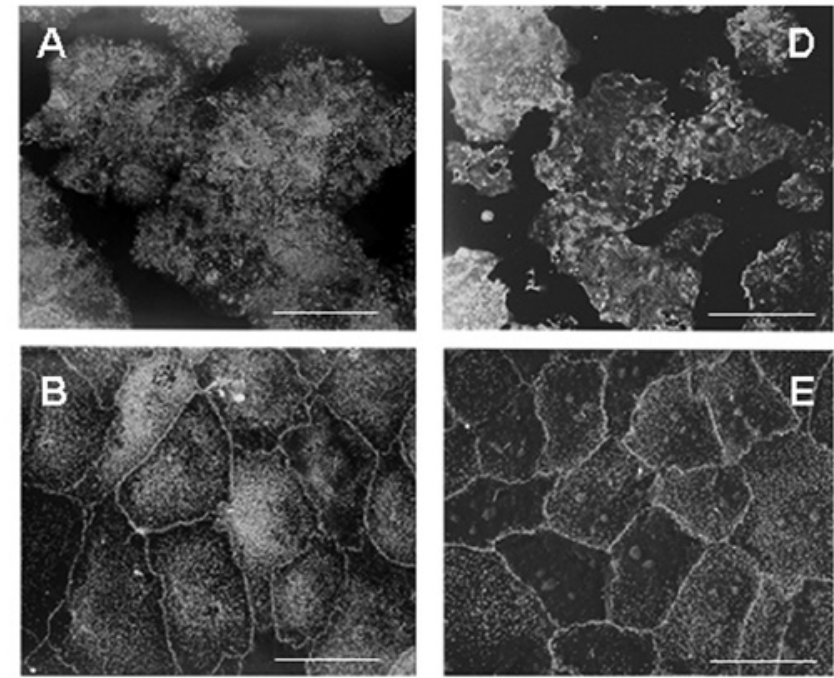

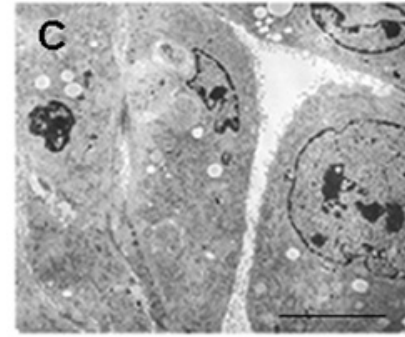

LC1

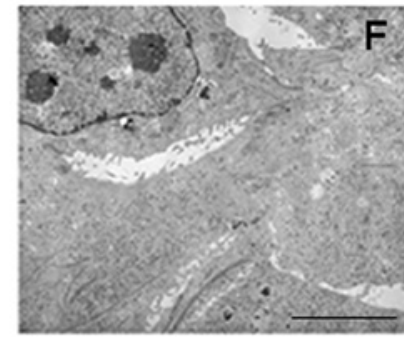

LC2
Figure 1. Electron microscopic analysis of primary cultured mouse lung adenocarcinoma cells. (A) and (D) Low magnification of LC1 and LC2 cells, respectively. (B) and (E) Scanning electron micrograph of LC1 and LC2 cells showing compacted, pavement-like cells with dense (LC1) and less dense (LC2) microvilli on the surface. (C) and (F) Transmission electron micrograph showing junction and microvilli of primary LC1 and LC2 cells. Bar, $75 \mu \mathrm{m}$ in (A) and (D); $30 \mu \mathrm{m}$ in (B) and (E); and $10 \mu \mathrm{m}$ in (C) and (F).

adherent primary cultures were successfully established for propagation in 2 out of 5 cases (40\%) of surgically obtained malignant tumours. Cells from 3 cases failed to passage, although the cells initially attached and started to form colonies in the first 2 weeks of culture. Tumour samples were considered to be moderately or well-differentiated. Notably, no correlation was found between histological grading or staging and success in establishing primary cultures. In all adherent cultures, cell migration from organoids was evident within 7 days of initiation, with 2 cultures proliferating for several months. Two primary cells (LC1 and LC2) were sub-cultured by mechanical scraping within 1-3 months of the initiation of cultures (Fig. 1A and D), whereas fibroblasts remain attached. LC1 and LC2 subcultures between passages 3 and 6 were used to check the epithelial nature of passaged cells by electron microscopy and by immunostaining of cytokeratin and $\beta$-catenin (Figs. 1 and 2).

Cellular morphology. In two primary cultures, cells grew in monolayers, though in varying degrees of attachment to the plastic support. Cells were polarized, exhibiting junctional complexes and microvilli, albeit with large differences in number and organisation even between cells in the same 
A

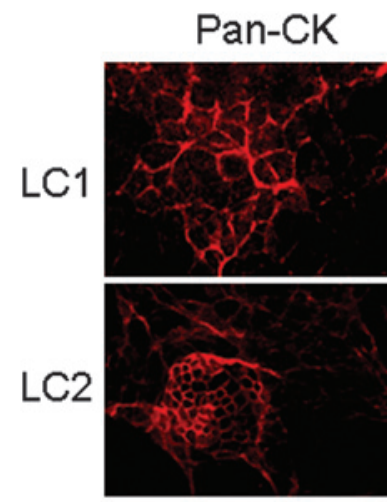

B

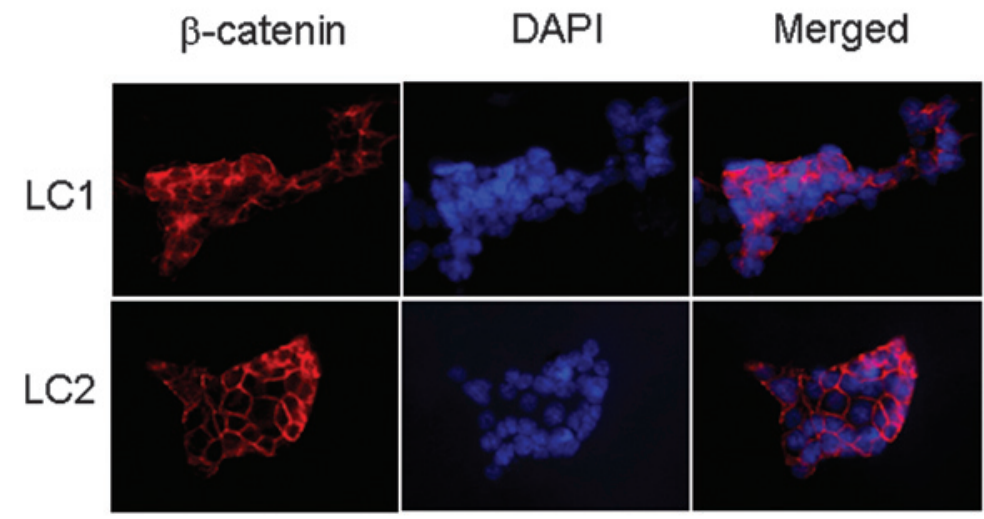

DAPI
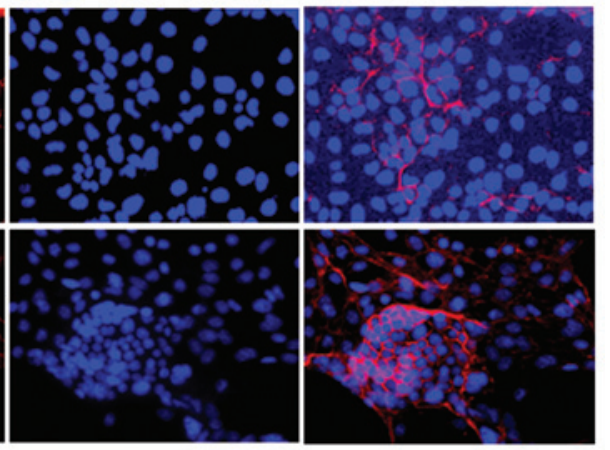

Merged

Figure 2. Epithelial nature of primary cultured mouse lung adenocarcinoma cells. (A) A heterogeneous positive pan-cytokeratin immunostaining for LC1 and LC2 cells, DAPI stained for nuclear content. (B) Positive $\beta$-catenin immunoactivity for LC1 and LC2 cells.

culture (Figs. 1 and 2). A scanning electron micrograph of a primary culture of a mouse lung carcinoma showed that the majority of polarized cuboidal cells were tightly packed and densely decorated at the apical aspect with well-developed microvilli (Fig. 1B). However, the same culture also contained a number of cells of an epithelial nature that exhibited few or no microvilli (Fig. 1E). A transmission electron micrograph of a primary culture of a mouse lung carcinoma showed a typical epithelial nature as visualized by their decoration to a greater or lesser extent with microvilli (Fig. 1C and F).

Epithelial characters of primary cultured cells were further evaluated using antibodies specific to epithelial markers. Immunocytochemical staining of primary cells revealed strong positive staining for pan-cytokeratin (Fig. 2A) and $\beta$-catenin (Fig. 2B). Cells appeared heterogeneous in size at the edge of the cell patch (Fig. 2), and various expression levels of pancytokeratin between cells in the same culture were frequently observed (Fig. 2A).

Primary cultured cells are tumourigenic. To evaluate whether primary cells were tumourigenic, LC1 or LC2 $\left(1 \times 10^{6}\right)$ cells per mouse were inoculated subcutaneously for two weeks. The mice developed tumours within two weeks, and tumour size was between 5 and $10 \mathrm{~mm}$ in diameter (Fig. 3A). Histological analysis revealed the inoculated solid tumours to be more or less dense, with compacted cells (Fig. 3B) expressing various levels of pan-cytokeratin and E-cadherin immunoactivity, indicating their epithelial origin.
$\mathbf{A}$
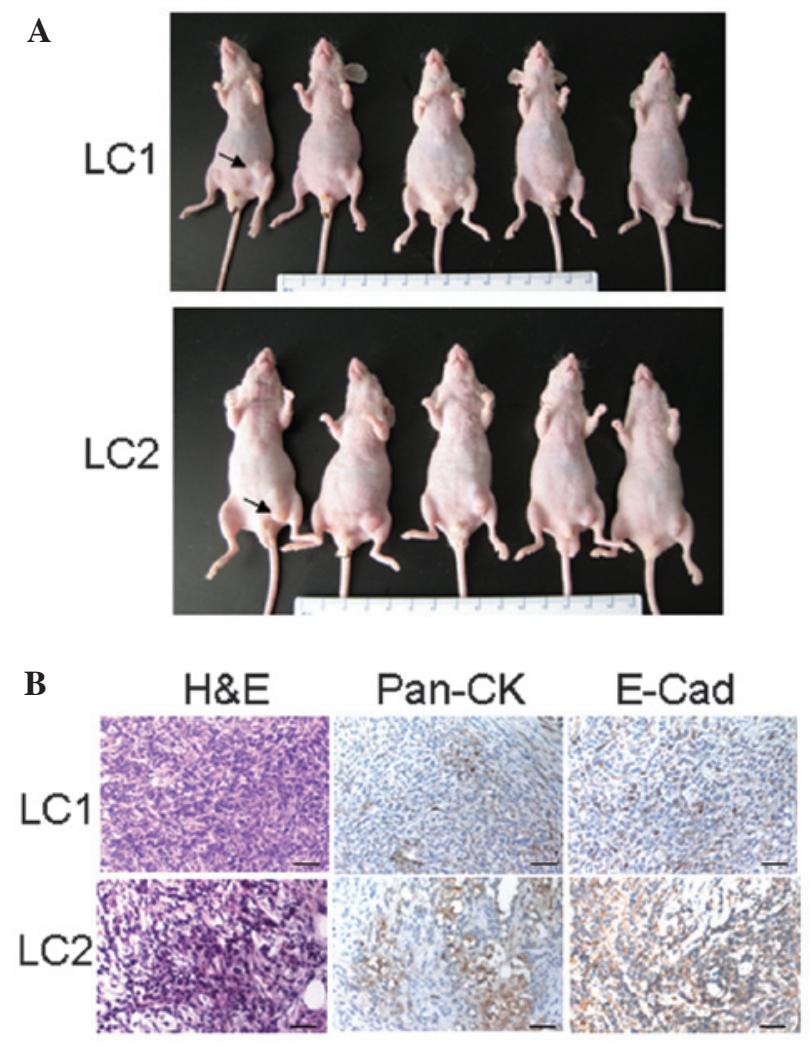

Figure 3. Tumourigenicity test. (A) Primary cells inoculated subcutaneously into the left inguinal of BALB/c nude mice for two weeks; tumours were detected in all mice (arrows). (B) Histological analysis of tumours and immunohistochemical staining for pan-cytokeratin and E-cadherin. Bar, $400 \mu \mathrm{m}$. 


\section{Discussion}

The majority of mouse lung tumours were well- or moderately differentiated adenocarcinomas utilized for the reproducibility and optimal establishment of primary cultures. Subsequently, short periods of enzymatic digestion and seeding at high density were used. Since fibroblast contamination presents severe difficulties in the establishment of primary cultures, various methods were adopted in this study in order to obtain cells of a purely epithelial nature. Mouse fibroblast feeder layers and fibroblast-conditioned media apparently supported the preferential growth of epithelial cells. Thus, our method of organoid isolation and selective cultivation of epithelial cells on glass coverslips in petri dishes with mouse fibroblast feeder cells protected against fibroblast overgrowth.

Primary cell growth results in the formation of colonies. For passage initiation, the advantages of mechanical scraping are two-fold. First, the cell sheets are released, allowing cells to attach to fresh culture plates more slowly than single cells. Second, replating the cell clumps a few hours after culture into fresh culture plates also protects against fibroblast contamination. Individual mouse lung adenocarcinomas exhibit pronounced regional phenotypic heterogeneity, resulting from the accumulation of specific genetic lesions in different parts of the same tumour.

Although various methods have been used in clinical anticancer drug screening (14-16), this study simplified the primary culture protocol and most of the primary cells were cultured and maintained for 2 weeks. Consequently, if the extent of responsiveness of a given tumour to a specific anti-cancer drug is studied in an in vitro model, it should reflect the cellular diversity of the tumour from which it was derived (11-12,17). In this respect, it is of note that the primary cultures described in the present study contain distinct sub-populations of epithelial cells. For instance, the density and the forms of microvilli on the apical plasma membrane were not uniform in a single culture. This may indicate differences in cellular functions as well as differentiation, and allow investigation of the mechanisms of DNA damage response in tumourigenesis (7).

Our results demonstrate the usefulness of the culture model employed in this study for further applications of personalized medicine of primary cultures of human lung adenocarcinoma cells in anti-cancer drug screening (18-20), and for the improvement of personalized drug response. Additionally, primary cultures may be useful in adjuvant therapy of human lung cancer.

\section{Acknowledgements}

This study was in part supported by grants from the Natural Science Foundation of China (No. 30870354), Project of Scientific Innovation and Creative for Jilin Provincial Oversea Scholars (No. 2010273), and Jilin Provincial Science \& Technology Services (No. 200805120 \& No. 20090732).

\section{References}

1. Stewart BW and Kleihues P (eds): World Cancer Report. IARC Press, Lyon, p182, 2003

2. Butnor KJ and Beasley MB: Resolving dilemmas in lung cancer staging and histologic typing. Arch Pathol Lab Med 131: 1014-1015, 2007.

3. Mulshine JL and Henschke CI: Prospects for lung-cancer screening. Lancet 355: 592-593, 2000.

4. Wright GS and Gruidl ME: Early detection and prevention of lung cancer. Curr Opin Oncol 12: 143-148, 2000.

5. Zhang X, Miao X, Liang G, et al: Polymorphisms in DNA base excision repair genes ADPRT and XRCC1 and risk of lung cancer. Cancer Res 65: 722-726, 2005.

6. Shao M, Ma H, Wang Y, et al: Polymorphisms in excision repair cross-complementing group 4 (ERCC4) and susceptibility to primary lung cancer in a Chinese Han population. Lung Cancer 60: 332-339, 2008.

7. Bartek J, Lukas J and Bartkova J: DNA damage response as an anti-cancer barrier: damage threshold and the concept of 'conditional haploinsufficiency'. Cell Cycle 6: 2344-2347, 2007.

8. Tong WM, Cortes U, Hande MP, et al: Synergistic role of Ku80 and poly(ADP-ribose) polymerase in suppressing chromosomal aberrations and liver cancer formation. Cancer Res 62: 6990-6996, 2002.

9. Tong WM, Yang YG, Cao WH, et al: Poly(ADP-ribose) polymerase-1 plays a role in suppressing mammary tumourigenesis in mice. Oncogene 26: 3857-3867, 2007.

10. Tong W-M, Hande MP, Lansdorp PM and Wang ZQ: DNA-strand break-sensing molecule PARP cooperates with p53 in telomere function, chromosomal stability and tumour suppression. Mol Cell Biol 21: 4046-4054, 2001

11. Wang CG, Sun M, Zhao XJ and Zhang XY: Effect of STAT3 siRNA-induced inhibition of STAT3 gene expression on the growth and apoptosis of Lewis lung cancer cells. Chin J Clin Oncol 3: 392-399, 2006.

12. Wang CG, Wang RY, Sun M, et al: In vivo antitumour effect of siRNA against STAT3 on transplanted Lewis lung cancer in mice. Chem Res Chin Univ 24: 322-329, 2008.

13. Tong WM, Ohgaki H, Huang H, Granier C, Kleihues P and Wang ZQ: Null mutation of DNA strand break-binding molecule poly(ADP-ribose) polymerase causes medulloblastomas in p53(-/-) mice. Am J Pathol 162: 343-352, 2003.

14. Yang S, Su J, Cao J, Zhang P, Lu J, and Xie W: Establishment of a novel Chinese human lung adenocarcinoma cell line CPA-Yang1 which produces highly bone metastases in immunodeficient mice. Zhongguo Fei Ai Za Zhi 12: 753-759, 2009.

15. Yaghi A, Zaman A and Dolovich M: Primary human bronchial epithelial cells grown from explants. J Vis Exp 26: pii: 1789, doi: $10.3791 / 1789$.

16. Kalinina T, Gungor C, Thieltges S, et al: Establishment and characterization of a new human pancreatic adenocarcinoma cell line with high metastatic potential to the lung. BMC Cancer 10: $295,2010$.

17. Higashiyama M, Oda K, Okami J, et al: Prediction of chemotherapeutic effect on postoperative recurrence by in vitro anticancer drug sensitivity testing in non-small cell lung cancer patients. Lung Cancer 68: 472-477, 2010.

18. Camps C, Sirera R, Iranzo V, Taron M and Rosell R: Gene expression and polymorphisms of DNA repair enzymes: cancer susceptibility and response to chemotherapy. Clin Lung Cancer 8: 369-375, 2007.

19. Hardwicke MA, Oleykowski CA, Plant R, et al: GSK1070916, a potent Aurora $\mathrm{B} / \mathrm{C}$ kinase inhibitor with broad antitumour activity in tissue culture cells and human tumour xenograft models. Mol Cancer Ther 8: 1808-1817, 2009.

20. Powell C, Mikropoulos C, Kaye SB, et al: Pre-clinical and clinical evaluation of PARP inhibitors as tumour-specific radiosensitisers. Cancer Treat Rev 36: 566-575, 2010. 